\title{
Technologies for the Scientific Exploration of the Outer Planets
}

\author{
By \\ Thomas R. Spilker, \\ Independent Consultant, Monrovia, CA 91016 \\ and \\ Patricia M. Beauchamp, \\ Jet Propulsion Laboratory/California Institute of Technology, Pasadena, CA 91109
}

Formal endorsements by

Jeff Bowman, UCSD Scripps Inst.; Scott Edgington, JPL/CIT; Alfred McKuen, U of Arizona LPL; Jeff Moore, NASA Ames Res. Ctr.; Carol Paty, U of Oregon; Abigail Rymer, JHU/APL; Britney Schmidt, Georgia Inst. of Tech.; Linda Spilker, JPL/CIT

\begin{abstract}
The outer solar system is a vast region populated by an extremely diverse collection of objects, from giant planets with their satellites, rings, magnetospheres to ocean worlds and dwarf planets, and a variety of smaller objects. Each has stories to tell about the origin and evolution of our solar system and the potential for extraterrestrial life. This extreme diversity results in the need for a wide range of technologies to effectively address the long list of high-priority science questions the outer solar system poses. The list of technologies that could greatly enhance the science value of space flight missions to outer planet systems is far too long to cover in this limited space, so this paper summarizes the benefits that technologies can bring to mission concepts, categorizes the different types of technologies appropriate to scientific exploration of the giant planets and their systems, and supplies references to sources that describe those technologies in much greater detail than is possible here. The OPAG Goals and Objectives document identifies a flagship-class ice giant mission as its number one priority for a new start in the decade 2023-2032. Although analyses for ice giant mission concepts have identified only one enabling technology needing development for those concepts, namely a radioisotope power source of appropriate size, efficiency, and lifetime, technology developments will play a large role in reducing the mass and power of multiple sciencecraft support systems. The mass and power savings allow more mass and power for science instruments and direct support systems such as telecommunications, increasing the mission's science value and easing the path to approval and flight. Thus it is likely that a suite of technological advances, rather than an individual technology, will enable a large ice giant mission. In general any technology is useful for missions if it provides mass savings, electric power savings, an increase in instrument or investigation performance, or an increase in the returned data volume. Landed ocean world probes and atmospheric entry probes involve some technologies that are specific to those investigations. A productive technology R\&D program needs a sufficient and consistent funding profile to maintain its continuity. NASA, via PESTO, should ensure a robust and well-funded
\end{abstract}


program of technology development to maintain a world-class program of outer planet exploration. 


\section{INTRODUCTION}

The outer solar system is a vast region populated by a diverse collection of objects, each with stories to tell about the origin and evolution of our solar system and the potential for extraterrestrial life. It is home to the giant planets and their systems of satellites, rings, magnetospheres, and other associated features. While the giant planets themselves are diverse - Jupiter is very different from Saturn, and both are very different from the ice giants Uranus and Neptune - the collection of satellites, rings, etc. is at least equally diverse, if not more. This extreme diversity results in the need for a wide range of technologies to effectively address the long list of highpriority science questions, described in the Goals Document of NASA's Outer Planets Assessment Group (OPAG) [1] and summarized in OPAG's umbrella science white paper to this Decadal Survey Committee [2].

Some of the technology needs are general, applicable to any type of platform. Others are more body- and mission-specific. Categories include flying by a giant planet system, orbiting a giant planet, entering an atmosphere, orbiting a satellite, landing on a satellite, and accessing the subsurface of a solid body.

The large heliocentric distances and large planetary masses of the giant planets result in technological challenges common to any mission of scientific exploration to those systems. For spacecraft that would orbit one of the outer solar system's planets these challenge areas include:

- Providing sufficient electric power to operate a scientifically worthwhile spacecraft

- Providing telecommunications sufficient to transfer the needed data volume

- Maintaining a tolerable thermal environment for spacecraft systems and components

- Providing sufficient propulsive capability to complete a mission, including orbit insertion

The first three of these also apply to flyby missions. Satellite orbiters include all four but place even more emphasis on propulsive capability. Additional challenges apply to atmospheric entry probes and landed missions to ocean worlds. For entry probes, they include:

- Surviving a relatively long post-deployment "coast" period without external power,

- Surviving the extreme hypersonic entry environment, and

- Operating over a large range of temperatures during descent through the planet's atmosphere

For landed ocean worlds missions, they include:

- Autonomous precision landing and hazard avoidance

- Subsurface access in cryogenic vacuum environments, and

- Subsurface-to-surface communications

Various forms of autonomy, such as autonomous science observation planning and execution and data prioritization and mining, are potentially greatly enhancing for ocean world missions. Pages 65-71 of the OPAG Goals Document [1], the Technologies section, includes a comprehensive list and descriptions of technologies critical or enhancing for ocean world exploration. There is also a detailed discussion of such technologies in the report of OPAG's Roadmap to Ocean Worlds group (ROW) [3].

Some of these challenges result in secondary challenges. For instance, the large heliocentric distances result in long telecommunications round-trip times and relatively low data rates that challenge the utility of platforms operating on limited lifetimes or with short on-station periods. 
Some events of great interest, e.g. a meteor impact on a satellite, occur on such short time scales that it is impossible to obtain specific observations of the event with the ground team in the command loop. Missions requiring rapid responses to various situations might benefit greatly from some level of autonomous operation, eliminating a round-trip light time (RTLT) of up to eight hours for communications and possibly much longer delays for ground-in-the-loop decisions.

Hardware solutions to any of these challenges, primary or secondary, are coupled: a solution to one impacts one or more of the others. A hardware solution generally adds mass and often requires electric power, immediately impacting the propulsion and electric power subsystems, and often impacting other basic spacecraft systems such as spacecraft structure and attitude control.

Note that a technology can be enabling, meaning that a mission is impractical without its level of performance and there is no alternative, or it could be enhancing, meaning that a mission is technically feasible without its performance level, but having it improves the mission's science return and thus its value. But the distinction between enabling and enhancing depends on a mission's requirements. For instance, a mission whose investigations require a data return rate of only $10 \mathrm{kbps}$ from Neptune could use existing telecom technologies, so high performance telecom would be considered enhancing. But for a more ambitious mission requiring a $100 \mathrm{kbps}$ rate, high performance telecom technology would be enabling. Providing science value sufficient to win approval for implementation might require a suite of technologies that, while individually not enabling, together become enabling because they achieve the threshold science value.

With one important exception, available technologies handle each of the major challenges listed above, with the caveat that achieving a threshold science value might require one or more enhancing technologies. The 2017 Ice Giants Pre-Decadal Mission Study Report [4], an excellent general reference, covers many of those technologies. The exception is an electric power source. NASA recently indefinitely delayed the eMMRTG development program. The MMRTG, the only unit currently available, is inadequate for many outer solar system missions, especially ones that would orbit an ice giant, OPAG's top mission priority for a new start in the decade 20232032 [2]. A program to develop an RPS appropriate for longer-duration outer solar system missions, and make it available in time for the launch opportunities, is clearly enabling. There are too many potentially useful enhancing technologies to list all here, but in general any technology is useful if it provides mass savings, power savings, an increase in instrument or investigation performance, or an increase in returned data volume. Instrument technologies are treated in a separate white paper so they will not be treated here.

\section{ELECTRIC POWER}

Electric power production is critical to any space mission. For outer solar system applications three performance metrics are cogent: lifetime, specific energy (total electric energy supplied over its lifetime normalized by the battery's mass), and specific power (the maximum electric power available normalized by battery mass). Figure 1 charts specific energy and specific power for various power sources. At Jupiter missions with modest power needs can use solar power, as demonstrated by the Juno mission, and at Saturn there might be some missions with very limited power needs that could use solar as their primary power source. Beyond Saturn a nuclear-based power source, specifically the radioisotope power source (RPS), currently is the only practical alternative. The requirement for new electric power technology has changed since release of NASA's 2016 Ice Giants Missions study [4]. In early 2020 NASA announced that the development of the eMMRTG, a lynchpin of those studies, had been indefinitely delayed. Since 
missions such as an ice giant orbital mission, performance of the MMRTG, the only RPS currently available, is insufficient for long-duration missions such as an ice giant orbital mission, an alternative is clearly enabling. NASA should properly fund and staff a program to develop an RPS appropriate for longer-duration outer solar system missions, including flagshipclass missions with their higher power requirements, and make it available in time for advantageous launch opportunities.

Use of an RPS assumes availability of sufficient nuclear fuel, which for NASA RTGs is ${ }^{238} \mathrm{Pu}$. That isotope is almost ideal for RPSs, but requires a dedicated production process to yield usable quantities. US civilian production of ${ }^{238} \mathrm{Pu}$ is under development with a production rate scheduled to increase to $1.5 \mathrm{~kg} /$ year by 2026, sized to meet NASA's estimated Planetary Mission Directorate needs. Those need estimates are based on a model of mission types and schedules that carries considerable uncertainty. The planetary science community should encourage NASA to adhere to its schedule of ${ }^{238} \mathrm{Pu}$ production capacity, and to investigate other potential sources should

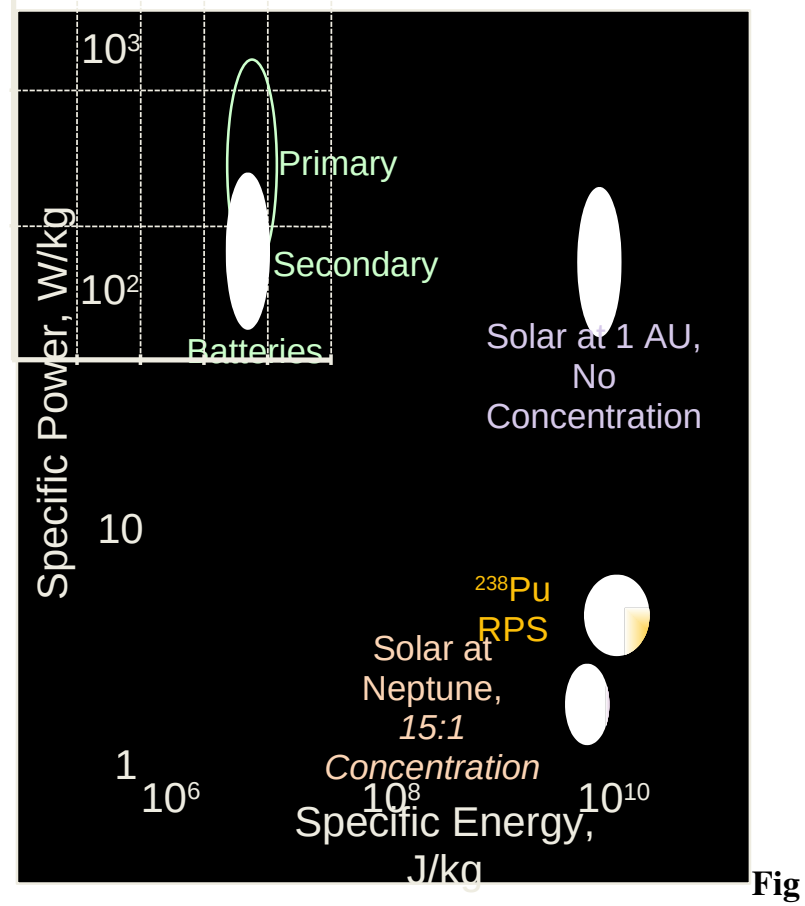

ure 1. Specific energy and specific power ranges of candidate primary and secondary power sources. the need arising from a high-priority mission opportunity, such as an ice giant flagship mission, exceed the modeled need profile. Spacecraft thermal systems share the need for ${ }^{238} \mathrm{Pu}$, since nuclear heat sources play an important part in reducing the electric power requirements of the thermal subsystem. This topic is treated in more detail in Section IV.

Use of solar power for an outer planet mission has been demonstrated by Juno. Many subsequent mission concepts, including some approved for implementation, such as ESA's JUICE and NASA's Europa Clipper, use solar power as their primary power source. Multiple Discovery and New Frontiers mission concepts, including some to the Saturn system with modest power requirements, use solar power, but beyond Saturn its use is problematic due to low solar intensity and 'LILT' effects. The ROW priorities, mission scenarios, and technologies document [5], page 20, describes the advantages, disadvantages and best applications of solar power for outer solar system missions.

In addition to a primary electric power source most scientific spacecraft need a secondary power source, and sometimes carry "daughter" spacecraft that require their own power supplies. For these purposes chemical batteries are important components. There are two types of chemical batteries currently available for flight: primary batteries that aren't rechargeable and are used as primary power sources, and secondary batteries that are rechargeable and are used for energy storage. Primary batteries are capable of somewhat higher specific power than secondary batteries, making them good choices for short-duration mission profiles requiring high power, such as atmospheric entry probes or short-lived landers. Secondary batteries can store energy 
from a primary power source and are critical for two important spacecraft scenarios: when an operating mode temporarily exceeds the power capacity of the primary power source, the secondary source provides the "load leveling" capacity to meet the increased need; and if the primary power source goes offline temporarily, the secondary source allows the spacecraft to "call for help" (implement safe mode) and continue functioning while the ground team diagnoses the problem and uplinks a fix. When infused into a specific mission, both types of battery have masses set by the mission's requirements: the higher the total energy required or the maximum power required, the larger the battery mass. Improvements in specific energy or specific power of battery systems reduce the mass of batteries a spacecraft must carry. Those mass savings ripple through the spacecraft systems, especially structure and propulsion, to yield greater savings, ultimately allowing more mass for science. Investments toward improving battery technologies are well worthwhile.

\section{PROPULSION}

Propulsion, the process of changing the translational momentum of an object, is the fundamental technology that makes space exploration possible. This paper describes three types of propulsion: chemical rocket propulsion, electric rocket propulsion, and aerodynamic propulsion. All three offer potential implementation options for outer planets missions, sometimes multiple type on a single mission. Astrodynamics software that performs wideranging trajectory searches can ease propulsion challenges. The better that software, the more flyable trajectories can be found, sometimes saving significant mass and mission duration.

Chemical rocket propulsion is a fairly mature technology: its current state enables orbital missions to every giant planet system. Advances are still being made, but in more indirect areas. During chemical rocketry's developmental period of the last century specific impulse $\left(I_{S P}\right.$, an alias for exhaust velocity) was a fertile area of $\mathrm{R} \& \mathrm{D}$. The higher is $I_{S P}$, the less propellant is required to handle a mission's required $\Delta V$, which is the velocity change the spacecraft's propulsion system must impart; seemingly it is the most direct avenue for improvement. But with this mature technology improvements in $I_{S P}$ are more incremental now, so other aspects offer greater potential for outer solar system missions. An example is development and infusion of low-freezing-point bipropellants that save spacecraft power and mass by requiring less heater power to prevent propellants from freezing in locations not easily kept warm by other means.

One sub-type of chemical propulsion, cold gas propulsion, is potentially useful for landings on airless ocean worlds, or any location with biological or pre-biological potential, due to its ability to avoid chemical contamination of the landing site. Such systems often use nitrogen $\left(\mathrm{N}_{2}\right)$ but other systems using propellants offering higher $I_{S P}$ would be advantageous.

Three different types of electric rocket propulsion (usually referred to simply as electric propulsion) are distinguished by their type of power supply. Solar electric propulsion (SEP) systems are powered by solar arrays; Radioisotope electric propulsion (REP) systems are powered by an RPS, as described in Section I; and nuclear electric propulsion systems are powered by a fission-reactor-based power source. Of the three, only SEP is sufficiently mature for use for projects starting in the decade 2023-2032, and for the foreseeable future is useful only in the inner solar system. For outer solar system missions this limits its utility to boosting spacecraft onto transfer trajectories to outer solar system destinations, but this is an important capability, especially for Neptune orbital missions. The primary impediment for REP is not the 
propulsion systems themselves but the relatively low specific power of existing and planned RPSs that power those systems.

Aerodynamic propulsion, or aeroassist, uses the aerodynamic forces of drag and/or lift arising from travel through a body's atmosphere to change a craft's momentum. There are four primary types: aerobraking, where a vehicle already orbiting a body makes multiple passes through the body's atmosphere, using drag to decrease the orbit's apoapsis, with each pass imparting a small change in velocity; atmospheric entry, where a vehicle enters a body's atmosphere and uses drag to effect a complete deceleration to subsonic velocities; aerocapture, where a vehicle approaching a body on an unbound (hyperbolic) orbit uses drag and possibly lift to decelerate to a bound orbit during a single pass through the atmosphere; and aerogravity assist, where a vehicle changes its momentum with respect to a second body by using drag and possibly lift during a pass through a third body's atmosphere. Aerobraking and atmospheric entry were demonstrated decades ago. Aerocapture has yet to be demonstrated, but a recent summary of the readiness state of aerocapture [5] indicates it is ready for implementation at less challenging destinations such as Titan or Mars, and subsequently might offer significant increases in mass inserted into orbit at Uranus or Neptune. Like aerocapture, aerogravity assist has not been demonstrated but potentially offers substantial mass savings. The white paper by Arnold et al. [6] suggests it is also ready for implementation at certain destinations such as Saturn/Titan.

\section{COMMUNICATIONS}

Science data are the end product of all science missions except sample return missions, and monitoring and commanding capabilities are critical to any practical mission, so telecommunications ('telecom') is second only to propulsion as a general enabler for space exploration. During six decades of deep space telecom experience many innovative technologies have been developed and successfully implemented, but the quest for more science data from longer distances drives the need for continued improvement in telecom capabilities. Notably, recent ice giant mission concept studies found that using current telecom technologies met threshold data return requirements $[4,7]$, but the science return would have benefitted from better performance. The OPAG Goals Document [1] and the ROW Group report [3] describe various opportunities for increasing telecom performance. In addition to system-wide upgrades in aspects such as signal coding and keying methods, on the spacecraft side of the telecom system performance is largely a result of the "power-aperture product": a system's supportable data rate is proportional to the transmitted power times the area (not diameter) of the antenna's primary reflector. Lowmass, deployable antennas and high-efficiency RF power amplifiers directly address this. Optical telecom is coming, but will not be ready for outer planet missions in the decade 2023-2032.

Atmospheric entry probe missions carry unique telecom requirements not addressed by spacecraft-to-Earth systems, but the technologies involved are mostly mature, so most applicable developments are engineering developments, not technology developments. But any technology that increases RF power amplifier efficiency or improves the specific power or specific energy of batteries will decrease the probe's mass, and that savings ripples through the entire flight system.

Ocean world missions also carry unique telecom requirements, and some needs will require technology development. Data relay from a subsurface element to a surface element involves signal propagation through an inhomogeneous, cryogenic, lossy solid medium, and possibly through a lossy liquid medium. Contact might be intermittent, and the autonomy to preserve data 
in the face of unpredictably intermittent contact will be important. The ROW Group report [3] describes various aspects of telecom performance for ocean world exploration.

\section{THERMAL}

Technologies for thermal control of spacecraft in the outer solar system are generally mature, but there are technologies that need to be maintained and potential enhancements. Methods for making better use of heat produced on a spacecraft can be enhancing by saving mass and precious electrical power. Methods for coupling waste heat into a spacecraft from an RPS have been demonstrated, but that assumes the presence of an RPS, which assumes the availability of an RPS of appropriate characteristics (lifetime, conversion efficiency, power output, etc.) and the ${ }^{238} \mathrm{Pu}$ to fuel it, as discussed in Section II. It is difficult to couple that waste heat to spacecraft components in locations remote from the bus interior, such as instruments on booms, and attitude control thrusters and their feed lines. Radioisotope Heater Units (RHUs) are effective for such needs and save electric power if they are available, and again that assumes availability of ${ }^{238} \mathrm{Pu}$ to fuel them. Availability of ${ }^{238} \mathrm{Pu}$ to cover all NASA mission needs is a critical issue.

\section{AUTONOMY}

Although aside from spacecraft self-preservation ("safe mode", etc.) autonomy is not an enabling technology in the strictest sense, some level of autonomous operation will be important for missions to the giant planets to implement science investigations well beyond the accomplishments of prior missions. In the case of ice giant missions and especially ocean world investigations at Triton, with 6 to 8 hour round-trip light times (RTLTs) impeding ground-in-theloop operations, autonomous operation will be vital for achieving some high-priority science objectives. Examples of very useful autonomous operations include:

- Science observation planning and execution

- Science data prioritization and mining

- Navigation and propulsive maneuver planning, design, and execution

- Telecom network control

- Landing technology, including precision landing and hazard avoidance

In addition to the algorithms needed to implement these capabilities, robotic hardware is needed to implement the algorithms' commanded activities, and there must be sufficient rad-hard compute capacity to handle the throughput requirements. The OPAG Goals Document [1] includes a thorough technology discussion on pages 65-71 with descriptions of many types of autonomy technologies applicable to all aspects of the scientific exploration of the outer planets. There is also a detailed discussion of technologies applicable to exploration of ocean worlds in the ROW report [3].

\section{ATMOSPHERIC ENTRY, DESCENT, AND LANDING}

Atmospheric entry, descent, and landing (EDL) technologies address the needs of atmospheric entry probes (EPs) and possibly ocean worlds landers at Titan and perhaps Triton. In addition to the enhancing telecom and electric power technology mentioned in Section III, EPs at the giant planets require high-performance thermal protection systems (TPSs) for the extreme hypersonic entry and deceleration phase. A series of white papers from the TPS group at NASA's Ames Research Center (ARC) address TPS and associated systems, notably the HEEET material now 
at TRL 6, for EPs and other atmospheric flight applications [6, 9, 10, 11]. EP descent modules that traverse a large vertical range in a giant planet atmosphere could experience temperatures ranging from cryogenic (as low as $50 \mathrm{~K}$ ) to Venus-like in a period about an hour, so thermal control technologies to mitigate those effects would be very useful. The ROW Group report [3] describes the need for EDL technologies for ocean world exploration, including autonomous precision landing and hazard avoidance. The range of EDL technologies needed covers everything from airless bodies such as Enceladus and Europa, to bodies with thin atmospheres such as Triton and Pluto, to Titan, with its very thick atmosphere, and gravitational accelerations that span more than an order of magnitude. Each of these bodies brings very different requirements.

\section{RECOMMENDATIONS}

NASA's Planetary Science Division now has its Planetary Exploration Science Technology Office (PESTO), that can focus on technologies for planetary science missions, including outer solar system missions. OPAG recommends that NASA invest in the technologies described in this paper and the referenced technology papers with sufficient and consistent funding levels. Developing the technologies is key, but the ability to test them in a relevant environment is also key. Developing appropriate testbeds for these technologies is an important component of a viable technology program.

\section{REFERENCES}

1. Moore, J., and the OPAG Steering Committee, Scientific Goals for Exploration of the Outer Solar System, available at https://www.lpi.usra.edu/opag/goals-08-28-19.pdf, August 2019.

2. Moore, J., Spilker, L., and the OPAG Steering Committee, Exploration Strategy for the Outer Planets 2023-2032: Goals and Priorities, white paper submitted to the Planetary Science and Astrobiology Decadal Survey 2023-2032, available at http://surveygizmoresponseuploads.s3.amazonaws.com/fileuploads/623127/5489366/20842376aad3ea240cf9a2790f91a462f7f_MooreJeffreyM.pdf, April 2020.

3. Hendrix, A.R., Hurford, T.A., et al., The NASA Roadmap to Ocean Worlds, Astrobiology 19 No. 1, https://doi.org/10.1089/ast.2018.1955 , Jan. 2019.

4. Hofstadter, M., Simon, A., Elliott, J., Reh, K., et al., Ice Giants Pre-Decadal Survey Mission Study Report, JPL document D-100520, available at https://www.lpi.usra.edu/icegiants/mission_study/, June 2017.

5. Spilker, T.R., Adler, M., Beauchamp, P.M, et al., A Qualitative Assessment of Aerocapture and Applications to Future Missions, Journal of Spacecraft and Rockets, Vol. 56, 1-10, 10.2514/1.A34056, Nov. 2018.

6. Arnold, J.O., Spilker, T.R., et al., Heatshields for Aerogravity Assist Vehicles Whose Deceleration at Titan Saves Mass for Future Flagship Class Exploration of Enceladus, white paper submitted to the Planetary Science and Astrobiology Decadal Survey 2023-2032, August 2020.

7. Rymer, A., A Large Strategic Class Mission Study for the Exploration of the Neptune-Triton System, oral presentation to the Planetary Mission Concept Studies Workshop, available at https://www.youtube.com/watch?v=1NrYIVNvqLI\&feature=youtu.be , May 2020.

8. Spilker, T.R., Enabling Technologies for Ice Giant Exploration, Phil. Trans. Roy. Soc. A, in press, accepted for publication Sep. 2020. 
9. Ellerby, D., Hwang, H, and Gasch, M., TPS and Entry Technologies for Future Outer Planet Exploration, white paper submitted to the Planetary Science and Astrobiology Decadal Survey 2023-2032, August 2020.

10. White, T., Ellerby, D., Gasch, M., et al., Thermal Protection System Materials for Sample Return Missions, white paper submitted to the Planetary Science and Astrobiology Decadal Survey 2023-2032, August 2020.

11. Santos, J.A.B., and Edquist, K.T., Entry, Descent, and Landing Instrumentation, white paper submitted to the Planetary Science and Astrobiology Decadal Survey 2023-2032, August 2020. 